\title{
Knowledge, attitudes and practices about leishmaniasis among cutaneous leishmaniasis patients in Aleppo, Syrian Arab Republic
}

\author{
N. Abazid, ${ }^{1}$ C. Jones ${ }^{7}$ and C.R. Davies ${ }^{7}$
}

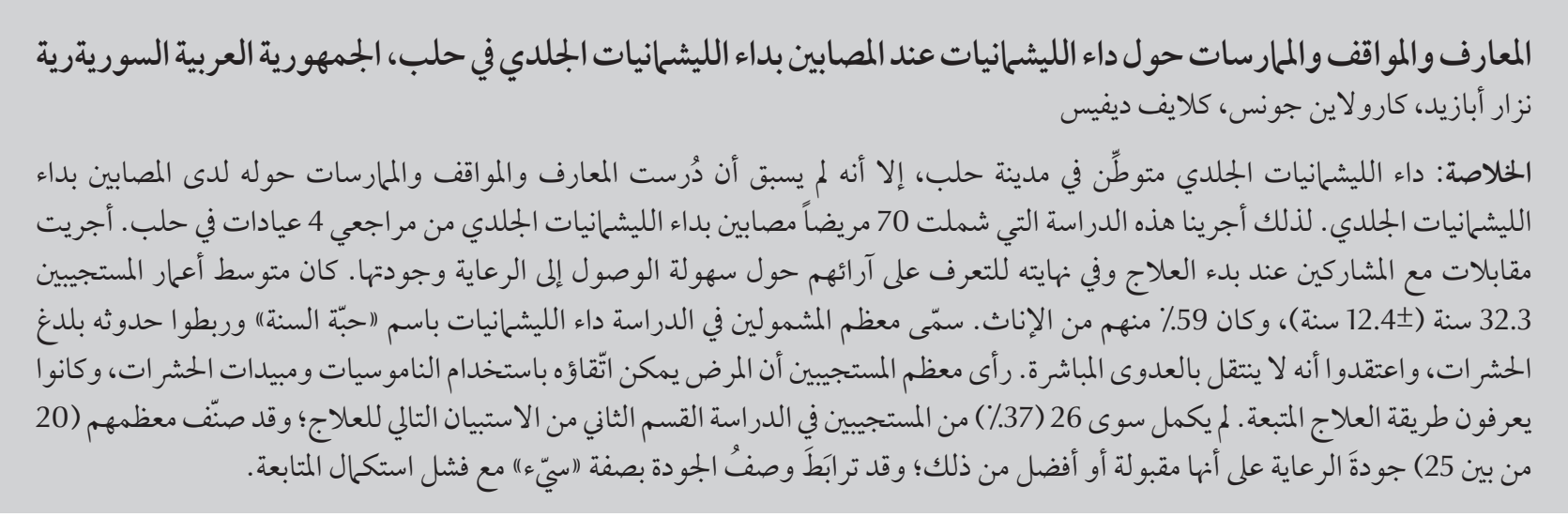

ABSTRACT Cutaneous leishmaniasis $(\mathrm{CL})$ is endemic in Aleppo and yet the knowledge, attitudes and practices (KAP) of CL patients in Aleppo has never been studied. We conducted a KAP study among $70 \mathrm{CL}$ patients attending 4 clinics in Aleppo. Participants were interviewed at recruitment and then at the end of treatment to ascertain their views on access to treatment and the quality of care provided. The mean age of respondents was 32.3 (SD 12.4) years and 59\% were female. Most respondents referred to the disease as "one-year sore", linked it to insect bites and believed it was not contagious. Most believed it was preventable by the use of bednets and insecticides and knew the treatment mode. Only 26 (37\%) respondents completed the second part of the questionnaire after treatment. Most of these $(80 \%)$ rated the quality of care as acceptable or better. Assessment of the quality as "bad" correlated with failure to complete follow-up.

Connaissances, attitudes et pratiques des patients atteints de leishmaniose cutanée à Alep (République arabe syrienne)

RÉSUMÉ La leishmaniose cutanée est endémique à Alep, mais les connaissances, les attitudes et les pratiques des patients atteints de cette affection n'ont jamais été étudiées. Nous avons mené une étude à ce sujet auprès de 70 patients dans quatre centres de santé à Alep. Les participants ont été interrogés à la sélection puis à la fin du traitement pour connaître leur opinion en matière d'accès aux soins et de qualité de ceux-ci. L'âge moyen des répondants était de 32,3 ans (E.T. 12,4 ans) et 59 \% étaient de sexe féminin. La majorité des répondants connaissait la maladie sous son appellation de "bouton d'un an », l'associait à des piqûres d'insectes et ne pensait pas qu'elle était contagieuse. La plupart d'entre eux croyaient qu'elle pouvait être prévenue par l'utilisation de moustiquaires et d'insecticides et avaient connaissance du mode de traitement. Seuls 26 répondants (37\%) ont rempli la seconde partie du questionnaire après le traitement. La plupart d'entre eux (80\%) déclaraient que la qualité des soins était acceptable, voire bonne. Lorsqu'elle était considérée comme « mauvaise », elle était corrélée à un échec de suivi après le traitement. 


\section{Introduction}

Cutaneous leishmaniasis (CL) has been endemic in Aleppo, Syrian Arab Republic for centuries, giving the disease one of its common names, Aleppo boil $[1,2]$. Aleppo is also the site of the first recognisable modern description of CL by Alexander Russell in 1756 [3], although the disease may have been described as early as in the 9th century under the name of Balkh sore [4]. Russell mentioned that the disease was known locally as habt il senne, which he translated as botch of a year.

The reported annual incidence of CL in Aleppo decreased in the 1950s, possibly due to insecticide spraying against malaria, but started to increase again in the late 1980s [5], from a few hundred during most of the 1980s to thousands since 1988 and to more than 10000 since 2003 [6].

Russell's observations suggest that CL has been recognised by the lay population since at least the middle of the 18 th century. However, to our knowledge, no studies have been published about the perceptions of the people of Aleppo concerning this common condition. Moreover, it seems that until quite recently there were no publications about popular perceptions of $\mathrm{CL}$ in the Old World, although anecdotal statements regarding these perceptions occasionally appeared in publications about the epidemiology or treatment of leishmaniasis. Siage, working in Damascus in the early 1960s, mentioned that the villagers tended to ignore the lesions because they are indolent, and "the scars are not usually feared" [7]. Peters, in his 1988 paper on CL in the Arabian Peninsula, mentions that this disease "was simply accepted as a fact of life" [8]. By contrast, in a report on the incidence of CL in Aleppo, Ashford et al. stated that, "Infection is most important as a public health problem in teenagers and young adults, for whom the cosmetic effects are often considered very serious" [9].
This latter suggestion is echoed by studies performed in Afghanistan, which report that $\mathrm{CL}$ is often considered stigmatizing. In Kabul, girls with scars are considered unsuitable for marriage, and it is considered inappropriate for women with active lesions to raise children, cook or wash for the family $[10,11]$. A later study in Kabul on the social impact of CL confirmed these previous observations [12]. Recently, a more in-depth study on community perceptions of CL was performed in Kabul province [13]. The results confirmed the stigmatizing perceptions of $\mathrm{CL}$ among the local population.

In Turkey, a study examined the psychological impact of CL in Sanliurfa, a region adjacent to Aleppo governorate [14]. The authors compared active CL patients with healthy controls and healed CL patients and found that active and healed patients showed higher anxiety and depression scores and lower body image satisfaction scores compared to controls. They also speculated about possible stigmatization but did so in the context of established relationships between conspicuous skin conditions and psychological disorders, i.e. not specifically related to CL. This seems to be the first and, to date, only study that addresses the psychological impact of Old World CL.

It is worth mentioning that all these studies were performed in areas where, as in Aleppo, the causative organism is believed to be Leishmania tropica and the cycle of transmission is anthroponotic.

An indirect indication of the dearth of published research about perceptions concerning $\mathrm{CL}$ in the Old World is the total absence of references to any sociobehavioural research publications about CL in the above-mentioned studies. Such knowledge may guide future health education efforts contributing to leishmaniasis control in Aleppo. The aim of this study therefore was to gain a better understanding of the knowledge, attitudes and practices (KAP) of CL sufferers and their carers regarding the disease in Aleppo.

\section{Methods}

\section{Study sample}

We carried out a cross-sectional questionnaire survey among a group of participants recruited from patients involved in a study of the clinical course, parasite sensitivity and treatment of CL in Aleppo between November 2006 and October 2007 (Abazid et al., in preparation). All participants initially recruited in the clinical study (or their care-givers if the patients were minors) were eligible for the questionnaire survey. However, it was not always possible to administer the questionnaires to all recruited patients because of the high number of patients in the leishmaniasis clinic. Some patients initially recruited to the clinical study and who answered the questionnaire were later excluded because they were negative by smear and culture. However, their questionnaires were included because a formal establishment of the diagnosis is not likely to affect people's knowledge about and attitudes towards the disease.

Eligibility criteria for the KAP survey included: 1) referral to one of the 4 primary health care (PHC) centres (2 located in Aleppo city and 2 in the countryside surrounding the city) where the clinical study was being conducted; 2 ) residence in the $\mathrm{PHC}$ centre catchment area and 3 ) having $\leq 5$ active lesions.

For patients $<14$ years of age the child's caretaker answered the questions. When 1 adult was accompanied by several children (under 14 years of age) from 1 family all suffering from CL, only 1 questionnaire was completed (using the responses of the adult). If more than 1 patient from the same family were adults each adult was interviewed separately and individual questionnaires were completed for each.

While there were some refusals to participate in the larger clinical study, of the people who consented to participate in the clinical study no one refused to take part in the KAP study. However, 
some respondents did not answer all of the questions. Consequently, the number of answers for each question is sometimes less than the total number of questionnaires.

\section{Data collection}

A 2-phase structured questionnaire with open-ended questions was developed for administration to CL patients or, where the patient was $<14$ years, to the caregiver who accompanied them to the clinic.

The first phase of the questionnaire was administered on recruitment and consisted of questions related to the respondents' knowledge, attitudes, expectations and common practices related to CL. Data were recorded on the age, sex and education of the respondent (either the patient him/herself or the carer of the patient). Education was recorded as "years in study", with the only qualitative value for "university degree".

The second phase, administered near or after the end of treatment was designed to ascertain the participants' views on the ease of accessing treatment and the quality of care provided. The questions included travel time to the health centre, waiting time within the health centre, the perceived attitudes of the health centre staff and an overall qualitative evaluation of service as very bad, bad, acceptable, good, very good. Patients who dropped out and could be contacted were also asked about the reasons of interruption of treatment.

While both phases of the questionnaire where administered in the environs of the clinic, the locations for interviews were purposefully selected to ensure that health centre staff were unable to hear the conversation.

\section{Ethical considerations}

The study protocol was approved by the Ethical Committee at the London School of Hygiene and Tropical Medicine as well as by the Scientific Research Committee of Damascus University Faculty of Medicine. Administrative permission to conduct the study was obtained from the Syrian Ministry of Health. Informed consent to participate in the study was obtained from the patient or, where the patient was $<14$ years of age, from the patient's caretaker.

\section{Analysis}

The unit of analysis in this study is questionnaire, also referred to by the words "respondent" and "case". In the analysis and reporting of results, the term "patient-respondent" refers to patients over 14 years who answered the questionnaire themselves, and the term "caretaker-respondent" to the responses of the adults accompanying patients with CL under 14 years of age.

The data were analysed with SPSS, version 16. $P<0.05$ was considered statistically significant.

\section{Results}

\section{Sample description}

There were 132 patients recruited for the clinical study ( 25 were later excluded because they were smear and culture negative). From these 132, 70 phase 1 questionnaires were available for analysis. Of these 70 respondents, 26 also completed phase 2 of the questionnaire. The 3 main reasons for drop-out were: 1) failing to catch up with the respondent during their last visit; 2) failure by the patient to turn up for post-cure follow-up and 3) patient drop-out from treatment before cure.

The mean age of the 70 respondents was 32.3 standard deviation (SD) 12.4 years, the median was 32 years and the interquartile range (IQR) was $22-40.25$ years. Forty-eight $(59 \%)$ of the respondents were female. There were 39 (56\%) patient-respondents and 31 (44\%) caretaker-respondents. The caretaker-respondent was mother in 23 cases (about three-quarters of caretaker-respondents); the father in 7 cases and the grandmother in 1 case. Patient-and caretaker-respondent characteristics are compared in Table 1 . The patient-respondents were significantly younger (mean 30.3 years, median 28 years) than caretaker-respondents (mean 35.2 years, median 35 years) (Mann-Whitney test, $P=0.039$ ) but there were no other significant differences between the groups for the other characteristics on which information was collected.

Data on education were available for 67 respondents. About one-third (22 respondents) reported 6 years of education. The mean number of years of education was 6.4 (SD 4.4) years, median $(\mathrm{IQR})=6(5-8)$ years. Of the 67 respondents, 13 were illiterate $(0$ years of education), 16 had $\geq 9$ years of education, 9 had finished secondary school ( 12 years) and 3 had a university degree.

\begin{tabular}{|c|c|c|c|}
\hline Variable & Patients $(n=39)$ & Caretaker $(n=31)$ & $P$-value \\
\hline & Mean (median) & Mean (median) & \\
\hline \multirow[t]{2}{*}{ Age (years) } & $30.3(28)$ & $35.2(35)$ & $0.039^{a}$ \\
\hline & No. (\%) & No. $(\%)$ & \\
\hline Sex & & & $0.366^{b}$ \\
\hline Male & $14(36)$ & $8(26)$ & \\
\hline Female & $25(64)$ & $23(74)$ & \\
\hline Education (years) & & & $0.946^{b}$ \\
\hline$\leq 6$ & $24(62)$ & $17(61)$ & \\
\hline$\geq 7$ & $15(38)$ & $11(39)$ & \\
\hline
\end{tabular}

${ }^{a}$ Mann-Whitney $U$ test.

bearson's $\chi^{2}$ test. 


\section{Knowledge}

\section{Disease name}

The majority of the respondents (55/68, 81\%) reported "one-year sore" as the only name for the disease; Two very close variants were encountered, habt es-saneh and habbayyet es-saneh. Three respondents reported Leishmania as the only name. Four respondents knew more than one name: one gave the answers one-year sore and Aleppo sore, one Aleppo sore and Leishmania, one one-year sore and Leishmania and one all 3 names. The respondent who reported 3 names had a university degree, and the 2 respondents who mentioned Aleppo sore each had 14 years of education. This may suggest that among those who took part in the study, alternative names such as Aleppo sore and Leishmania were only used by those participants with the higher levels of education.

There was no difference in naming the disease by sex. By educational level, $37 / 41$ (90\%) with $\leq 6$ years of education mentioned one-year sore while only $16 / 24(67 \%)$ with $\geq 7$ years named it this $\left(P=0.042\right.$, Pearson $\chi^{2}$ test $)$.

\section{Sources and mode of transmission and contagiousness}

Responses about the possible causes of the disease were available for 66 respondents, 32 of whom suggested that there was more than 1 possible disease source. The responses were ranked by frequency and differences were analysed by sex and education. The sources of disease mentioned are presented in Table 2 ranked by frequency of mention. In all groups baqq (sandflies) were mentioned most frequently as the cause of the disease. The insects most commonly identified (by 31 respondents) as being responsible for disease causation were baqq and barghash, the local names usually applied to tiny biting flying insects, such as sandflies. Five of these 31 respondents also mentioned flies as the causative agent. Mosquitoes were implicated by 13 respondents, 4 of whom also implicated flies and 1 sandflies. In addition, 5 respondents named unspecified insects and 4 mentioned flies only.

Insects were the most frequently mentioned disease source (55 respondents) with most of these respondents (48) suggesting that insect bites were the mechanism for causing the disease. Six respondents specified that the insects transmitted the disease from polluted sources, such as polluted water or wells (3 respondents) and "dirt" (3 respondents) and 4 said that the disease was transmitted from person to person by insect vectors.

Twelve respondents reported microbes as the cause of the disease but only 1 named Leishmania as the causative agent. Eight respondents said the disease comes from dirt or dust, either by insects (3 respondents) or direct contact ( 2 respondents). The rest $(3 / 8)$ did not explain the transmission mode. Water as the cause/ source of disease was mentioned by 7 respondents; 3 believed the disease resulted from drinking-water, 2 said it was transmitted from water by insects. The other 2 respondents suggested that swimming or washing, i.e. direct contact was the cause/source of the disease.

Fifty-seven respondents provided their opinions about disease contagiousness, i.e. direct person-to-person transmission. Of these, 31 said that the disease was not contagious, 10 said that it was and 8 said that they did not know. Of the 10 who said the disease was contagious, 6 believed the disease could be transmitted either by direct contact (3 respondents) or indirect transmission through sharing objects (2 respondents), 4 did not specify the mode of transmission and 1 confused contagiousness with indirect (or direct) contact with insect vectors.

\section{Prevention, recurrence and time to healing}

Sixty-six respondents replied to the question on whether or not the disease could be prevented; of these 41 believed the disease could be prevented, 9 believed it could not and 16 did not know.

The participants who believed that the disease could be prevented suggested several ways in which this could be achieved. Some of the respondents provided more than 1 method of prevention while others did not specify how prevention might be achieved. The most commonly mentioned modes of prevention are shown in Table 3 .

\begin{tabular}{|c|c|c|c|c|}
\hline \multirow[t]{3}{*}{ Variable } & \multicolumn{4}{|c|}{ Cause (no. of responses) } \\
\hline & \multicolumn{4}{|c|}{ Rank } \\
\hline & 1 & 2 & 3 & 4 \\
\hline \multicolumn{5}{|l|}{ Sex } \\
\hline Male $(n=19)$ & Sandflies (8) & Mosquitoes (5) & Water (4) & Microbes (4) \\
\hline Female $(n=47)$ & Sandflies (25) & Mosquitoes (10) & Man (10) & Flies (10) \\
\hline \multicolumn{5}{|l|}{ Education (years) } \\
\hline$\leq 6(n=40)$ & Sandflies (22) & Flies (10) & $\operatorname{Man}(7)$ & Microbes (6) \\
\hline$\geq 7(n=25)$ & Sandflies (10) & Mosquitoes (8) & Microbes (6) & Man (4) \\
\hline
\end{tabular}

Respondents could give more than one answer in this category. 
Eight respondents suggested that the use of bednets and insecticides were effective prevention measures, but no other combination of responses was mentioned by more than 1 person.

Of 65 respondents, 42 (65\%) believed the disease might affect the same individual more than once, 12 (18\%) believed the disease never recurred and 11 (17\%) did not know.

The participants were asked about their perceptions on how long it took to cure the disease. Fifty-eight provided their opinions on time to healing and of these 42 respondents mentioned a specified period of time. The mean time to healing from these 42 responses was 11.25 (SD 6.3) months and the median (IQR) was 12 (6-12.75) months. Twenty respondents said the lesion needed 1 year to heal, 13 said they did not know how long it would take and 3 respondents mentioned that the disease does not heal unless treated.

\section{Disease recognition and treatment seeking}

In the patient-respondent group the lesion was first noticed by the patients themselves in 33 of 39 cases, undefined relatives in 3 cases, mother in 2 cases and daughters in 1 case. In the caretaker-respondent group the lesions were usually first noticed by the mother (in 23 cases), both parents in 3 cases and the father in 1 case. In only 4 cases was it the child him/herself who had noticed the lesion.

The most frequently mentioned action taken after the lesion was noticed was applying an antiseptic to the sore (25 cases). In 19 of these cases the antiseptic was described as spirto, a common reference to ethanol, iodine was used in 2 cases and an unspecified antiseptic in 4 cases. The second most frequently mentioned action was to do nothing (19), although 3 of these respondents specifically mentioned that they avoided touching the lesion. Thirteen respondents said that they had applied a cream or ointment (unspecified) and all other actions mentioned were only mentioned by one individual.

Time from first noticing the lesion to seeking treatment from a PHC centre was reported by 69 respondents and ranged from immediately to 1 year. The mean delay in treatment-seeking at a PHC among these participants was 2.4 (SD 2.1) months, and the median (IQR) was 2 (1-3) months.

The decision to treat the disease among patient-respondents was taken by the patients themselves in 30 of 39 cases, the patient's mother in 6 cases, husband in 2 cases and father in 1. Among caretaker-respondents, mothers took the decision in 18 of 31 cases, fathers in 11 cases and both parents in 2 cases.

Twenty-six of 39 of the patientrespondents came unaccompanied on their first visit to the PHC, 7 were accompanied by their mother, 3 by their husband, and 1 each by grandmother, wife or brother. The children in the study were accompanied by mothers in 23 of 31 cases and fathers in 6 cases.

\section{Expectations}

Replies about what the respondents knew about and expected from the treatment were available from 67 respondents.

\section{Nature of treatment}

Four respondents did not know what type of treatment to expect but the remaining 63 expected to receive injections. Of these 63 respondents, 54 said the injections are made into the lesions, 3 said they thought that the disease could be treated either intralesionally or intramuscularly, and 6 said they knew the treatment was by injections but did not know the mode of administration.

\section{Treatment duration}

When asked about the duration of treatment, 49 respondents provided a quantitative response. The expected duration ranged from 3 weeks to 1 year. The mean expected treatment duration was 3.2 (SD 3.3) months and the median (IQR), 2 (1-4.5) months. Ten respondents reported having no idea about the length of treatment, and 8 replied that it could not be predicted because it depended on the course of the disease.

\section{Cure recognition}

When asked about how they would know when they were cured, most of the 67 respondents (44/67) replied it would be based on their own criteria, either that the lesions would disappear (29 replies) or diminish (15 replies). However, 21 respondents said that cure would be determined by health

\begin{tabular}{|c|c|c|c|c|}
\hline \multirow[t]{3}{*}{ Variable } & \multicolumn{4}{|c|}{ Prevention measure (no. of responses) } \\
\hline & \multicolumn{4}{|c|}{ Rank } \\
\hline & 1 & 2 & 3 & 4 \\
\hline Complete sample & Bednets (17) & Insecticides (14) & Personal hygiene (9) & Waste disposal (5) \\
\hline Males & Personal hygiene (3) & Window mesh (3) & Sterilize water (2) & Waste disposal (2) \\
\hline$\leq 6$ years education & Insecticides (10) & Bednets (9) & Personal hygiene (6) & Waste disposal (3) \\
\hline
\end{tabular}

${ }^{a}$ There were 2 responses for waste disposal from persons with > 6 years of education. This group also gave 2 responses for each of window mesh and wash fruits. The responses offemales and those with $>6$ years of education had the same order as in the complete sample. 
centre staff and 2 replied that they did not know. Interestingly, the data from the second-phase questionnaire suggest that the proportion of those who continued their treatment until formal dismissal was the same among those who said cure was to be determined by health centre staff, 8 of 21 cases, and those who suggested their own criteria, 17 of 44 cases.

\section{Attitudes}

The most frequently mentioned reason for seeking treatment was to prevent disfigurement (23 cases). Other reasons related to limiting the size or spread of the lesions: to speed cure and to avoid multiplication of lesions ( 9 replies for each); to avoid lesion growth (8 cases); and to prevent the development of a deep ulcer (6 cases). In light of these responses it is perhaps not surprising that most respondents mentioned the appearance of the lesion (48 cases), followed by the permanent mark it is known to leave (23 cases) as the most unpleasant manifestations of the disease.

\section{Quality of care appraisal}

Only 26 respondents completed second phase of the questionnaire after completion of treatment. The majority of the participants (19 of 26 respondents) reported a travel time of $15 \mathrm{~min}$ utes or less. A further 2 respondents rated the travel time as "short".

In terms of waiting time at the clinic, 10 respondents only provided qualitative estimates, for example, long (3 respondents), medium (1 respondent) or short (6 respondents). Among the remaining 16 respondents the median waiting time was 10 minutes. Four respondents said they did not have to wait at all but waiting times between 45 minutes and $2 \frac{1}{2}$ hours were reported in 5 cases. These prolonged waiting times (and 2 of 3 reports of "long" waiting times) were in 2 specific clinics (1 urban and 1 rural) both of which were usually remarkably crowded.

Data on the quality of service were provided by 25 respondents, 11 of whom were from 1 urban centre and the rest distributed evenly between the other 3 centres ( 4 or 5 cases each). The results are presented in Table 4. Two of lower-than-acceptable appraisals were associated with the crowds at 1 of the urban PHC centres (and led to drop-out) and the other 3 related to perceived staff arrogance and lack of care in the other urban and 1 rural centre.

When we compared the respondents who did not complete follow-up until complete cure to those who were followed up until cure (Table 4), the responses of the former (10 respondents) were distributed more or less evenly between very bad and good, but none rated the services as very good. On the other hand, all 15 respondents who completed follow-up rated the services as good or very good. The median scores, with score of 1 corresponding to very bad and 5 to very good, were 2.5 in the former group and 4 in the latter $(P<$ 0.001, Mann-Whitney U test).

Only 6/43 drop-outs could be contacted. Two of them said they had left because the service was awful, 1 thought the treatment was inefficient, 1 considered the lesion had improved enough to stop treatment, 1 was busy during health centre opening hours, and 1 had moved away.

\section{Discussion}

To our knowledge, this is the first attempt to describe the knowledge, attitudes, expectations and practices associated with CL among CL patients (or their caregivers for children under 14 years of age) in Aleppo, Syrian Arab Republic.

The disease was widely recognised by the participants in this study and was referred to by its traditional name, habbt il senne, or "one-year pimple", a name that was first documented more than 250 years ago [3]. This name is almost identical to the one reported from Afghanistan, saldana [13], salek in the Islamic Republic of Iran [4] and from Russia, godovik [15], all of which incorporate year in their meaning.

First recognition of the appearance of the disease and the need to seek treatment from a PHC was by the patient themselves, or in the case of children < 14 years of age, by one or both parents (most commonly the mother).

Most of the respondents mentioned insects as factors associated with disease causation: either direct cause or by a vector. A little under half of the respondents implicated sandflies and nearly one-quarter implicated mosquitoes. While we did not explicitly ask the respondents to describe the insects that they mentioned, none of the respondents used the term sheikh saket, or "silent old man", that was used in a health education brochure as the popular name for sandflies in Aleppo. The second most commonly mentioned source of the disease was man, followed by "dirt" and water. These data are similar to

\begin{tabular}{|c|c|c|c|c|c|c|}
\hline \multirow[t]{2}{*}{ Cure status } & \multicolumn{6}{|c|}{ Service quality assessment (no. of cases) } \\
\hline & Very bad & Bad & Acceptable & Good & Very good & Total \\
\hline Followed up until cure & 0 & 0 & 0 & 11 & 4 & 15 \\
\hline Cure not documented & 3 & 2 & 3 & 2 & 0 & 10 \\
\hline Total & 3 & 2 & 3 & 13 & 4 & 25 \\
\hline
\end{tabular}


those found in the Istalif study [13] where insects, sandflies and mosquitoes, were perceived as the most common cause, followed by contagion, then environmental factors. In that same study, about half of the respondents implicated sandflies but only 10 participants mentioned bites as the mode of transmission [13], compared with 47 of 52 in our sample.

In the Istalif study several options of home treatment for CL are described [13]. Unfortunately, because of the structure of the questionnaire and the mode of recruitment of participants (patients who were already accessing the PHC seeking biomedical treatment) we do not have comparable data on possible options of home treatment from CL from this project. However, the data do show that among these participants at least, knowledge of the appropriate biomedical treatment regime (a series of injections) was high.

With regard to prevention measures, the most frequently mentioned was standard bednets, followed by insecticide spraying and then personal hygiene. Participants with a lower educational level more frequently placed insecticides before bednets and men were much more likely to mention personal hygiene first (Table 3). In the Istalif study [13] bednets and insecticides were also the 2 most frequently mentioned options, with the former most often mentioned by women and the latter by men.

The most unpleasant reported manifestation of CL in our sample was the appearance of the lesion and fear of a permanent mark. While in Afghanistan both active lesions and scars are associated with stigma [13], nothing of this kind was specifically mentioned by our patients and nor were they asked about it. However, the fact that the major concern for CL patients (or their parents in the case of children) was the fear of a permanent mark, suggest that some stigma may be attached to such scarring. This requires further investigation.

The design of this study does not allow us to make inferences about careseeking behaviour for CL among the population in general. However, the data suggest that among those who sought care for CL, the participants who maintained regular visits to the health centres in Aleppo were those for whom access was easy, with travel time of less than 15 minutes in about $80 \%$ of cases. It is possible that one of the main factors influencing continued and regular visits to the clinic was difficulty in accessing the services. This possibility merits further investigation.

This study was not designed as an in-depth investigation into beliefs and behaviours relating to CL but it has provided a first indication of the way in which CL is perceived in Aleppo. However, because participants were recruited from those in a broader clinical project, the study has several limitations, both in terms of the information collected and the generalizability of the findings. First, our respondents were recruited in the health centres where they came to seek diagnosis and treatment of leishmaniasis. For this reason, it is not surprising that they all believed leishmaniasis should be treated; those who may believe otherwise would obviously not be identified by this study. The sample is not representative of the entire population, but it does provide some useful insights into perceptions and attitudes towards the disease.

The questionnaire did not address issues of stigma and did not contain specific questions about possible marginalization, although most probably, if any significant social exclusion/isolation existed, it would have been mentioned in one way or another. We also did not explore the signs or symptoms that had precipitated treatment seeking.

The data on travel and waiting time and on perceived quality of care in our sample is likely to have been biased due to the high drop-out rate and, as mentioned previously, this issue requires further investigation. Few of the drop-outs could be contacted so the results from their responses must be interpreted with caution. However, the few data available do suggest that patient compliance is probably related to perceived quality of service, given the fact that half of the patients who did not complete the treatment rated the services bad or very bad and none rated them very good. On the other hand, all the patients who completed treatment rated them good or very good (Table 4). The attitude of our respondents to cure recognition (by health-centre staff as opposed to improvement of the lesion as assessed by the patient) was not predictive of treatment completion until cure.

Overall the findings from this study provide a glimpse into the knowledge, attitudes and practices of CL sufferers and their carers in Aleppo. Many of the results correspond closely with findings from other preliminary studies in Afghanistan and Turkey. However, none of the published studies to date provide a comprehensive understanding of how $C L$ is viewed and responded to by the broader community, or how the health providers themselves view and treat CL patients, or how accessibility and perceptions of quality of care affect treatment-seeking behaviour. These are all important areas for further in-depth investigation if any attempt is to be made to curtail the increase in incidence of this disease.

\section{Acknowledgements}

This article is dedicated to the memory of Professor Clive Davies who died on 3 March 2009. This study was supported by Wellcome Trust grant GR078078MA. NA was supported by a study grant from Damascus University.

This work formed part of a PhD thesis of N. Abazid submitted to the University of London. 


\section{References}

1. Canaan T. The oriental boil: An epidemiological study in Palestine. Transactions of the Royal Society of Tropical Medicine and Hygiene, 1929, 23:89-94.

2. Hovnanian P, Jebejian R, Yenikomshian HA. Dermal leishmaniasis in a newly inhabited section of Aleppo. Transactions of the Royal Society of Tropical Medicine and Hygiene, 1937, 31:191-192.

3. Russell A. The natural history of Aleppo, and parts adjacent. London, Printed for A. Millar, 1856:262-266 (Facsimile of 1756 ed.).

4. Elgood C. The early history of the Baghdad boil. Journal of the Royal Asiatic Society, 1934, 3:519-533.

5. Jalouk $\mathrm{L}$ et al. Insecticide-treated bednets to prevent anthroponotic cutaneous leishmaniasis in Aleppo Governorate, Syria: results from two trials. Transactions of the Royal Society of Tropical Medicine and Hygiene, 2007, 101(4):360-367.

6. Cutaneous leishmaniasis: Why are you neglecting me? Geneva, World Health Organization, 2007. (http://www.who.int/ neglected_diseases/resources/CutaneousLeishmaniasis.pdf, accessed 4 December 2011).

7. Siage J. La leishmaniose cutanée en Syrie [Cutaneous leishmaniasis in Syria]. Révue Medicale du Moyen-Orient, 1964, 21:445-452.

8. Peters W. "The little sister" - a tale of Arabia. Transactions of the Royal Society of Tropical Medicine and Hygiene, 1988, 82:179-184.
9. Ashford RW et al. Evidence for a long-term increase in the incidence of Leishmania tropica in Aleppo, Syria. Transactions of the Royal Society of Tropical Medicine and Hygiene, 1993, 87:247-249.

10. Reithinger $\mathrm{R}$ et al. Anthroponotic cutaneous leishmaniasis, Kabul, Afghanistan. Emerging Infectious Diseases, 2003, 9:727-729.

11. Reyburn $\mathrm{H}$ et al. The prolonged epidemic of anthroponotic cutaneous leishmaniasis in Kabul, Afghanistan: "bringing down the neighbourhood". Transactions of the Royal Society of Tropical Medicine and Hygiene, 2003, 97:170-176.

12. Reithinger R et al. Social impact of leishmaniasis, Afghanistan. Emerging Infectious Diseases, 2005, 11:634-636.

13. Stewart CC, Brieger WR. Community views on cutaneous leishmaniasis in Istalif, Afghanistan: implications for treatment and prevention. International Quarterly of Community Health Education, 2009, 29:123-142.

14. Yanık M et al. The psychological impact of cutaneous leishmaniasis. Clinical and Experimental Dermatology, 2004, 29:464467.

15. Borovsky PF. On Sart sore. Voenno-meditsinskĭ zhurnal, 1898, 195(11):925-941. Cited from Hoare CA Early discoveries regarding the parasite of oriental sore. Transactions of the Royal Society of Tropical Medicine and Hygiene, 1938, 32:67-92. 